\title{
BMJ Open Towards a middle-range theory of 'Stability of home-based care arrangements for people living with dementia' (SoCA-Dem): findings from a meta-study on mixed research
}

To cite: Köhler K, Dreyer J, Hochgraeber I, et al. Towards a middle-range theory of 'Stability of home-based care arrangements for people living with dementia' (SoCA-Dem): findings from a meta-study on mixed research. BMJ Open 2021;11:e042515. doi:10.1136/ bmjopen-2020-042515

\section{- Prepublication history} and additional supplemental materials for this paper is available online. To view these files, please visit the journal online (http://dx.doi.org/10. 1136/bmjopen-2020-042515).

Received 08 July 2020 Revised 16 December 2020 Accepted 24 March 2021

Check for updates

(C) Author(s) (or their employer(s)) 2021. Re-use permitted under CC BY-NC. No commercial re-use. See rights and permissions. Published by BMJ.

${ }^{1}$ German Centre for Neurodegenerative Diseases (DZNE), Witten, Germany ${ }^{2}$ Faculty of Health, Department of Nursing Science, Witten/ Herdecke University (UW/H), Witten, Germany

${ }^{3}$ Department of Health Services Research, School of Medicine and Health Sciences, University of Oldenburg, Oldenburg, Germany

Correspondence to

Kerstin Köhler;

kerstin.koehler@dzne.de

\section{ABSTRACT}

Background Most people with dementia and their informal carers live at home and strive to create a stable care situation for as long as possible. This preference of dyads is consistent with the global policy of ageing in place. Therefore, we aimed to develop a middle-range theory of stability guided by two research questions: How is stability of homebased care arrangements for people living with dementia constituted? What are the essential factors influencing stability?

Methods Within the 'Stability of home-based care arrangements for people living with dementia' project (SoCA project) at the German Center for Neurodegenerative Diseases (DZNE), we conducted a meta-study on mixed research. The analytical steps of meta-data analysis, meta-method and meta-theory are merged in an integrative synthesis. Eligible publications were identified through systematic database searches (MEDLINE, CINAHL and PsycINFO; last searched on 3 January 2017), backward/forward citation tracking and snowballing. All publications were screened against predefined inclusion criteria and evaluated through a quality appraisal. The analytical approach was thematic synthesis.

Results 99 publications were included. The middlerange theory conceptualises stability as a complex phenomenon comprising three components including eight concepts that are dynamically inter-related. The conceptual model visualises: (1) the trajectory of the dementia care arrangement, which involves a cyclic process of change and balancing over time; (2) the characteristics of the care arrangement, including needs, the carer role, the dyadic relationship and resources; and (3) the context, which is determined by society and culture and the respective healthcare system. The relevance of each concept in relation to stability changes over time. The forming of each concept is actively shaped by the informal carer.

Discussion This middle-range theory provides a thorough understanding of the stability of home-based care

\section{Strengths and limitations of this study}

- We developed a middle-range theory (SoCA-Dem theory) that provides an innovative conceptualisation of the phenomenon 'Stability of home-based care arrangements for people living with dementia'

- The SoCA-Dem theory can guide future research and policy development and can also be used to improve health and social care practice.

- Currently, we elaborate the interplay of the concepts that constitute 'stability' on a descriptive level: causal relationships need to be explored in subsequent empirical research.

- To better understand the complexity of home-based care arrangements, the predominant perspective of the informal carer within the SoCA-Dem theory should be complemented by the perspective of the person living with dementia and the diverse informal and professional supporters.

- This meta-study is based primarily on research from Europe and North America, which might limit the applicability of the SoCA-Dem theory in societies with different cultural contexts.

arrangements for people living with dementia and can be used to guide future research and practice.

Other This meta-study was funded by the DZNE and registered in PROSPERO (registration number CRD42016041727).

\section{INTRODUCTION}

Worldwide, most people with dementia live at home. ${ }^{1}$ It is the explicit wish of most people with dementia to stay in their familiar environments, ${ }^{2}$ and most informal carers wish to support the person with dementia at home for as long as possible. ${ }^{34}$ Driven by this motivation, informal carers are a vital resource for people living with dementia at home and play a crucial role in supporting dementia care in 
the community. ${ }^{5}$ In current research, policy and practice, these preferences are mirrored in programmatic agendas, such as 'ageing in place ${ }^{6}$ and reflected by an increasing focus on 'social health'. 7 These agendas emphasise the capability of people with dementia, their families and their social networks and aim to create prerequisites to enable dyads to live well with dementia in the places of their choice. ${ }^{8}$

This dyadic perspective can be complemented by the perspective of the social security and healthcare system. In light of demographic changes and the constantly growing prevalence of dementia worldwide, ${ }^{9}$ societies are challenged to provide adequate health and social care for an increasing number of ageing individuals and, thus, need to appropriately address limited financial resources. As dementia is among the strongest predictors of costintensive institutional care,$^{10}$ the support of home-based care has been discussed as an option to reduce expenses. ${ }^{11}$

The stability of home-based care arrangements is an important goal for people living with dementia and their informal carers and reflected by current social security and healthcare policy. Accordingly, the term 'stability' is used as a buzz word in research ${ }^{12}$ and policy ${ }^{13}$ contexts, but a thorough definition of stability is usually not provided. Often, stability and instability are recognised as opposing phenomena and are operationalised in a binary way. Thus, the transition of a person with dementia from home to an institution is often understood as a demarcation line between stability and instability. The time until institutionalisation greatly varies, and numerous studies have uncovered the predictors and risk factors of institutionalisation, ${ }^{14}$ but these studies did not focus on the process that finally leads to the termination of home-based care. Other studies have illuminated the interactions between individuals involved in home-based care ${ }^{15}$ but these interactions were not clearly linked to the phenomenon of stability or instability. In our understanding, continuing to live at home does not necessarily indicate a stable situation, and in the worst case, the needs of the involved persons can be neglected, leading to an exhausting situation for the person with dementia or the informal carer. ${ }^{16}{ }^{17}$ In contrast, living in a nursing home can be perceived as a satisfying and stable living situation. At first glance, the prevalent binary conceptualisation of stability versus instability based on the criterion of institutionalisation is appealing, but at a second glance, this conceptualisation does not capture the complexity of the phenomenon.

The significance of the interactions between all persons involved in a home-based dementia care arrangement and the central importance of the needs of these persons are highlighted in a published working definition of stability (see figure 1). ${ }^{18}$ Here, stability is defined as a dynamic process that unfolds over the trajectory of dementia care that is intentionally shaped by the actors and can be achieved only if the needs of the person with dementia and the informal carer are addressed. This definition is an essential part of the foundation of this
Stability of care arrangements for people with dementia living at home means

that through their (self-)caring actions, the people involved succeed in

adapting to the ever-changing care requirements so that the needs of the

person with dementia and his/her primary carer(s) are addressed

appropriately and care can continue at home.

Creating and maintaining stability is thus a dynamic process in which crises

are mastered and successful care routines are established. It also means that

a care arrangement moves on a continuum between stability and instability.

The termination of a home-based care arrangement and the transition into a different housing situation may result from a basically stable care situation or may be the result of a crisis that cannot be mastered.

Figure 1 Definition of stability of home-based care arrangements for people living with dementia. ${ }^{18}$

meta-study. It reflects the work conducted by Corbin and Strauss ${ }^{19}$ regarding managing chronic illness, Nolan and colleagues $^{20}$ regarding family care and von Kutzleben ${ }^{21}$ regarding informal dementia care trajectories, all of which conceptualise care as a process of vibrant interactions between individuals over time.

The working definition and this meta-study are integral parts of the 'Stability of home-based care arrangements for people living with dementia' project (SoCA project) at the German Center for Neurodegenerative Diseases (DZNE), site Witten, Germany. The SoCA project strives to develop a sound theory-based understanding of the complex phenomenon of stability and further explore the newly developed middle-range theory by conducting subsequent empirical studies using qualitative and quantitative research methodologies to finally identify and develop stability promoting interventions and care structures.

\section{AIM}

The aim of this study was to develop a middle-range theory of the 'Stability of home-based care arrangements for people living with dementia'. The theory generation process was driven by the following research questions: How is stability of home-based care arrangements for people living with dementia constituted? What are the essential factors influencing stability?

\section{METHODOLOGY}

\section{Study design}

We aimed to generate theory from published research findings. ${ }^{223}$ For this purpose, we performed a meta-study according to the approach described by Paterson and colleagues $^{24}$ and adapted the original meta-study methodology for the integration of mixed research by including qualitative and quantitative, mixed-method studies, as well as systematic reviews. During the theory development process, we considered the recommendations by Liehr and Smith, ${ }^{25} \mathrm{Im}^{23}$ and Risjord ${ }^{26}$ regarding the creation of middle-range theories. In the subsequent sections, we provide a very brief description of our methodology. The 


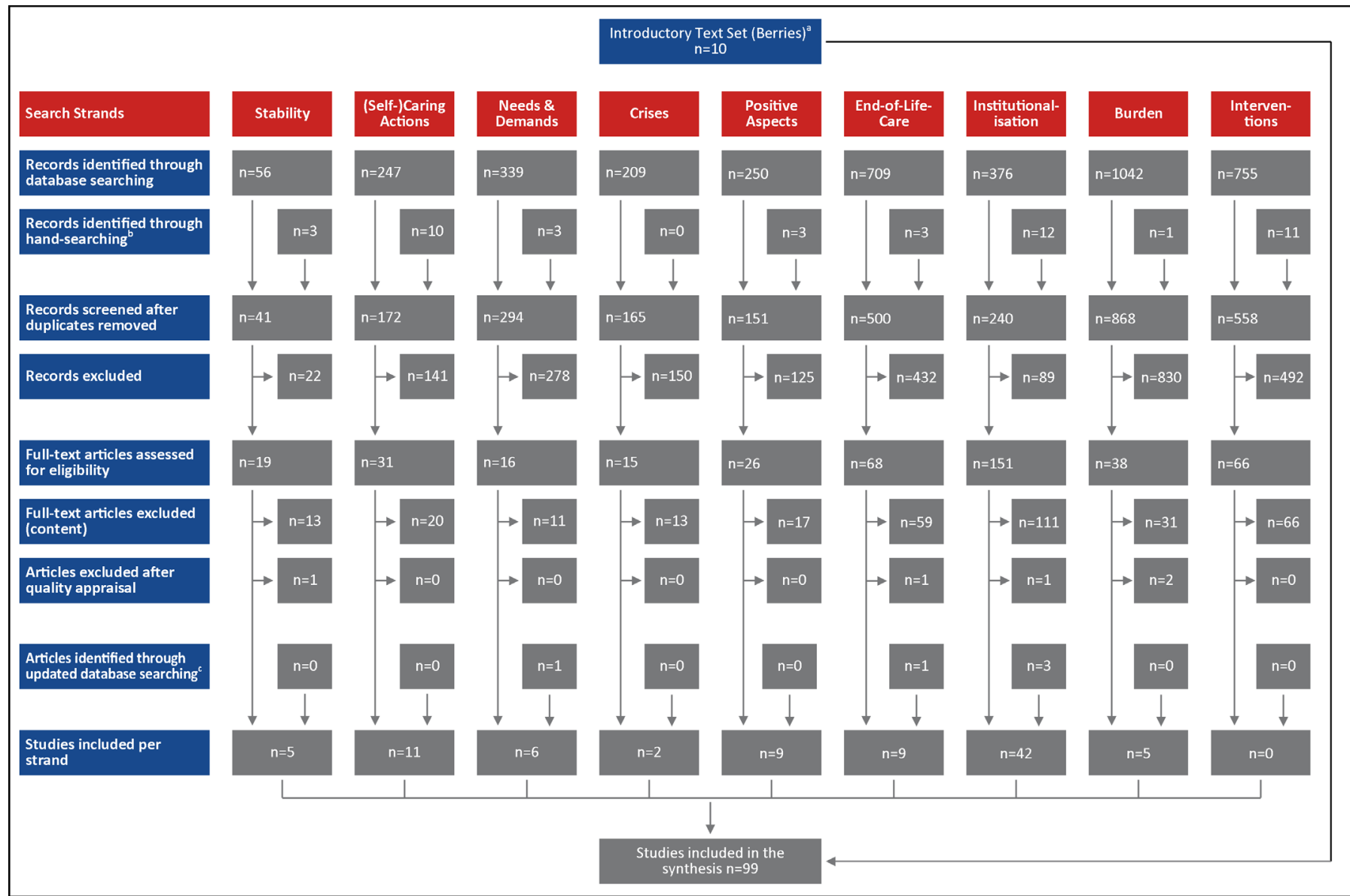

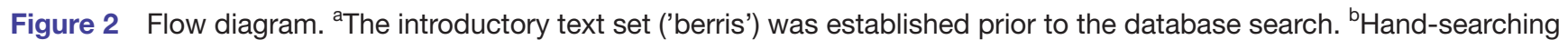
included backward citation tracking and snowballing. ${ }^{~}$ Updated databases search followed the same process as the initial search with the removel of duplicates, screening and assessment for eligibility.

details of the methodology were published in the respective study protocol. ${ }^{27}$ This meta-study is registered at the International Prospective Register of Systematic Reviews (PROSPERO registration number CRD42016041727). The reporting of this publication follows the recommendations in the ENTREQ statement. ${ }^{28}$

\section{Literature search}

Following the framework described by Boell and CecezKecmanovic, ${ }^{29} 30$ we searched for literature in openended 'hermeneutic loops', including the repeated steps of searching, sorting, selecting, acquiring, reading, identifying and refining. In parts, the cyclic literature search process was performed simultaneously with the ongoing data analysis: the final goal of the whole theory building process was to sufficiently encircle our phenomenon of interest by reaching conceptual saturation. ${ }^{31}$ The following search techniques were combined: berry picking, ${ }^{32}$ database searches, backward and forward citation tracking and snowballing. The database searches were performed between 24 September 2015 and 16 November 2015 in MEDLINE, CINAHL and PsycINFO and updated again on 3 January 2017 (referred to as 'updated database searching' in figure 2). Since the phenomenon of stability is rarely investigated directly, we used our previously published definition of stability ${ }^{18}$ to draw from it nine separate search strands and nine syntaxes. The complete syntaxes of the database searches are shown in online supplemental appendix A. During the iterative cyclic process of searching and analysing, we included further publications identified through citation tracking and snowballing.

\section{Screening process}

The titles and abstracts of all identified studies were screened by four authors (KK, JD, IH and MvK) in alternating teams of two authors. Full-text screening was performed by one author, who discussed his or her decision with at least one colleague. If disagreement occurred, consensus was reached by discussion. EndNote X7 was used to manage the screening process.

\section{Eligibility criteria}

The original meta-study methodology was designed for the synthesis of qualitative research only. In contrast, we decided to include all types of empirical research and (systematic) reviews. The rationale for this decision has been described elsewhere. ${ }^{27}$ The inclusion criteria are shown in online supplemental appendix B. We included 
publications written in English, German or French; no time limits were set.

\section{Quality appraisal}

We applied the quality appraisal recommended by DixonWoods and colleagues, ${ }^{33}$ which includes five criteria to identify studies with major methodological deficits (online supplemental appendix B). The decision to include or exclude a publication was made based on this quality appraisal and the publication's contribution to the proposed middle-range theory. Each paper was assessed by one researcher. If he or she was in doubt of the methodological quality, he or she discussed the paper with at least one other researcher until consensus was reached.

\section{Analysis and data collection process}

A meta-study combines the following three analytical steps, leading to a final meta-synthesis: meta-theory, meta-method and meta-data analyses. During our iterative analytical process, these steps were considered in an integrated process rather than individually. The insights gained from one analytical step immediately shaped the subsequent analyses. All analyses were performed using the Professional Research Software for Qualitative, Quantitative and Mixed Methods Research (MAXQDA 2018). The coding of the data was performed in teams of at least two researchers. As Thomas and Harden ${ }^{34}$ translated the widespread method of thematic analysis for the use in systematic reviews, we chose this analysis approach.

\section{Meta-theory}

Meta-theory involves a critical exploration of the theoretical frameworks that guided the included research. ${ }^{24}$ For each publication, we analysed the stated research gaps, aim(s), research question(s), explicitly stated theoretical frameworks and/or underlying concepts.

\section{Meta-method}

Meta-method analyses consider how the application of research methodologies influences the findings and shapes the current understanding of the phenomenon under research. ${ }^{24}$ For this purpose, we analysed the study design, methodology, sample, sampling, setting, data collection, data analysis and standardised measures used in each publication.

\section{Meta-data analysis}

A meta-data analysis is a comparative analysis of the findings of all included studies; this analysis contributes to the creation of an integrated body of knowledge. ${ }^{24}$ In our extended meta-study approach that included mixed research, we 'qualitised' the quantitative data. Our aim was to understand which concepts applied in quantitative studies are associated with 'stability' and how. Following Pope and colleagues ${ }^{35}$ and Sandelowski and colleagues, ${ }^{36}$ our main approach for 'qualitising' quantitative data was to extract relevant concepts from the texts, tables and figures of included quantitative studies, mixed-methods studies and systematic reviews and to compare these concepts across all studies. Concepts applied in quantitative studies often gave indications of an association with (in-) stability, which was examined in depth in qualitative studies. For details on 'qualitising', see also Dreyer and colleagues. ${ }^{27}$ We deduced a preliminary coding scheme based on our working definition. ${ }^{18}$ Following Thomas and Harden, ${ }^{34}$ we extended this coding scheme and analysed the findings of the included studies in three stages. We started the 'line-by-line coding' with a small introductory text set (figure 2). Gradually, the code system grew by induction, and the new codes were applied for each publication that was added over time while going back and forth in iterative loops of searching and analysing literature. In several coding rounds, the codes were translated to and compared across all publications of the meta-study sample. We organised these codes into 'descriptive themes', and we created major 'analytical themes' that formed the components and concepts of the proposed middle-range theory. Finally, no additional codes emerged from the added studies, and conceptual saturation was reached. ${ }^{31}$

\section{Meta-synthesis}

In the meta-synthesis, we elaborated on the relationship between the analytical themes. While we engaged more deeply with the material, at a certain point, the emerging middle-range theory no longer substantially changed. To ensure that the meta-synthesis was complete, we sampled a selection of publications from our sample and reviewed these publications again to determine whether the statements were all consistent with the status quo of the middle-range theory or any statements that might change the theory were overlooked. Our middle-range theory is visualised in a conceptual model that displays the interplay among the components and concepts in an organised form.

\section{Patient and public involvement}

Patients or the public were not involved in this meta-study.

\section{RESULTS}

\section{Sample description}

We included 99 publications in our meta-study. The detailed process of searching and selecting eligible publications is documented in the flow diagram in figure 2. During the screening process, 5 publications were excluded during the quality appraisal. During the analysis phase, the whole 'interventions' search strand was excluded from the sample because the content of the retrieved intervention reviews and studies did not contribute to the intended theory generation. A statement explaining the reasons for this decision is provided in online supplemental appendix C. Detailed information of each publication is provided in online supplemental appendix D, and a complete bibliography of the included papers is displayed in online supplemental appendix E. 
The included publications were authored by researchers in North America (the USA: $n=31$ and Canada: $n=12$ ) and Europe (Germany: $n=12$, the UK: $n=11$, the Netherlands: $\mathrm{n}=8$ and other European countries: $\mathrm{n}=12$ ); members of international consortia $(\mathrm{n}=8)$; and researchers in Asia or Australia $(\mathrm{n}=5)$; however, no publications authored by researchers in Africa or South America were included. The publications cover a wide range of scientific fields, including geriatrics, geriatric psychiatry, gerontology, dementia, nursing, palliative care, social sciences and so on. More than half of the included publications $(n=56)$ were published from 2010 onwards, 26 were published between 2000 and 2010, 13 were published in the 1990s and 4 were published in the 1980s.

50 included studies used quantitative methodologies, and 31 studies used qualitative methodologies. Furthermore, we included 7 mixed-methods studies and 11 systematic reviews (3 synthesised qualitative studies and 9 synthesised quantitative studies). 54 publications reported studies involving cross-sectional designs, and 34 publications reported studies involving longitudinal designs.

Only 21 publications explicitly stated the theoretical framework guiding their research process. Thereby, stress theories and stress models were chosen the most frequently $(\mathrm{n}=10)$. In 78 publications, no explicit theoretical framework was indicated, but the dominance of a stress/burden perspective was also apparent in these publications. For instance, 71 publications in our sample refer to the work conducted by Zarit et al, ${ }^{37}$ Pearlin et $a l^{38}$ and/or Aneshensel $e t a l^{39}$ who are researchers that advanced the understanding of stress and burden in caregiving. In the discussion section of our article, this conceptual bias and its impact on the development of our middle-range theory are reflected.

\section{Components and concepts of the middle-range theory and their interplay}

In this meta-study, we developed a middle-range theory of the 'Stability of home-based care arrangements for people living with dementia' (called the SoCA-Dem theory) that emphasises the perspective of the informal carer.

The current focus on the informal carer perspective in this middle-range theory is driven by our own previous work concerning informal dementia care trajectories, which identified stability as a guiding principle of informal carers. ${ }^{21}$ Therefore, we created a search strategy in which the presence of an informal carer in the home-based care arrangement was mandatory. ${ }^{27}$ As a consequence, most identified studies placed the informal carer at the centre of the research. Nevertheless, we are keenly aware that the perspectives of all actors (including the person living with dementia) in care arrangements are meaningful and shape the respective care arrangement, and we reflect on this conceptual bias in the discussion section of this publication.

We illustrate the middle-range theory in a conceptual model. The model visualises three components comprising eight concepts that altogether constitute stability of home-based care arrangements for people living with dementia from the perspective of the informal carer (see figure 3 ).

- Component 1 - trajectory: according to our understanding and consistent with Corbin, ${ }^{40}$ a chronic illness trajectory refers to the course of an illness/ condition over time and the actions that shape that course. The arrow at the bottom visualises the time course of the trajectory of a home-based care arrangement that covers the time span from the onset of the care arrangement to its termination. Above the arrow, a cyclic process displays the events and actions that shape the trajectory. This cyclic process comprises the two concepts of change and balancing and reruns in many loops over the trajectory.

- Component 2 - characteristics: four concepts, namely, needs, carer role, dyadic relationship and resources, and the forming of these concepts characterise the stability of a home-based care arrangement.

- Component 3 - context: the trajectory of a homebased care arrangement and its characteristics are influenced by the context, which includes the two concepts society and culture and the healthcare system.

The components and concepts of this middle-range theory are related in a dynamic interplay and cannot be interpreted in isolation from each other. Each component and concept is entwined with the other components and concepts, and all components and concepts influence and are influenced by the other components and concepts.

At any time in the trajectory, change may occur and trigger an action (balancing) of the informal carer. This balancing influences whether the needs of the person with dementia and the informal carer are successfully met or remain unmet. Balancing influences the informal carer's experience of the carer role, the development of the dyadic relationship and the management of resources. In contrast, met or unmet needs, the specific experiences of the informal carer, the development of the dyadic relationship and the management of resources may enable or hinder the informal carer from balancing in such a way that supports the stability of the care arrangement. In addition, this interplay between change and balancing over time and the characteristics of the care arrangement is influenced by the societal and cultural background and the respective healthcare system.

Thus, the forming of each concept is variable and change happens over the course of time, here over the care trajectory; each concept can assume a forming that promotes the stability of the home-based care arrangement or a forming that may challenge the stability. In addition, a specific concept in a specific form may have a minor impact on stability at the beginning of the trajectory but a major influence during the late phases and vice versa. At any time, there can be stable and instable phases.

These mechanisms of the dynamic interplay between the components and concepts of the SoCA-Dem theory are further clarified in the subsequent paragraphs, which 


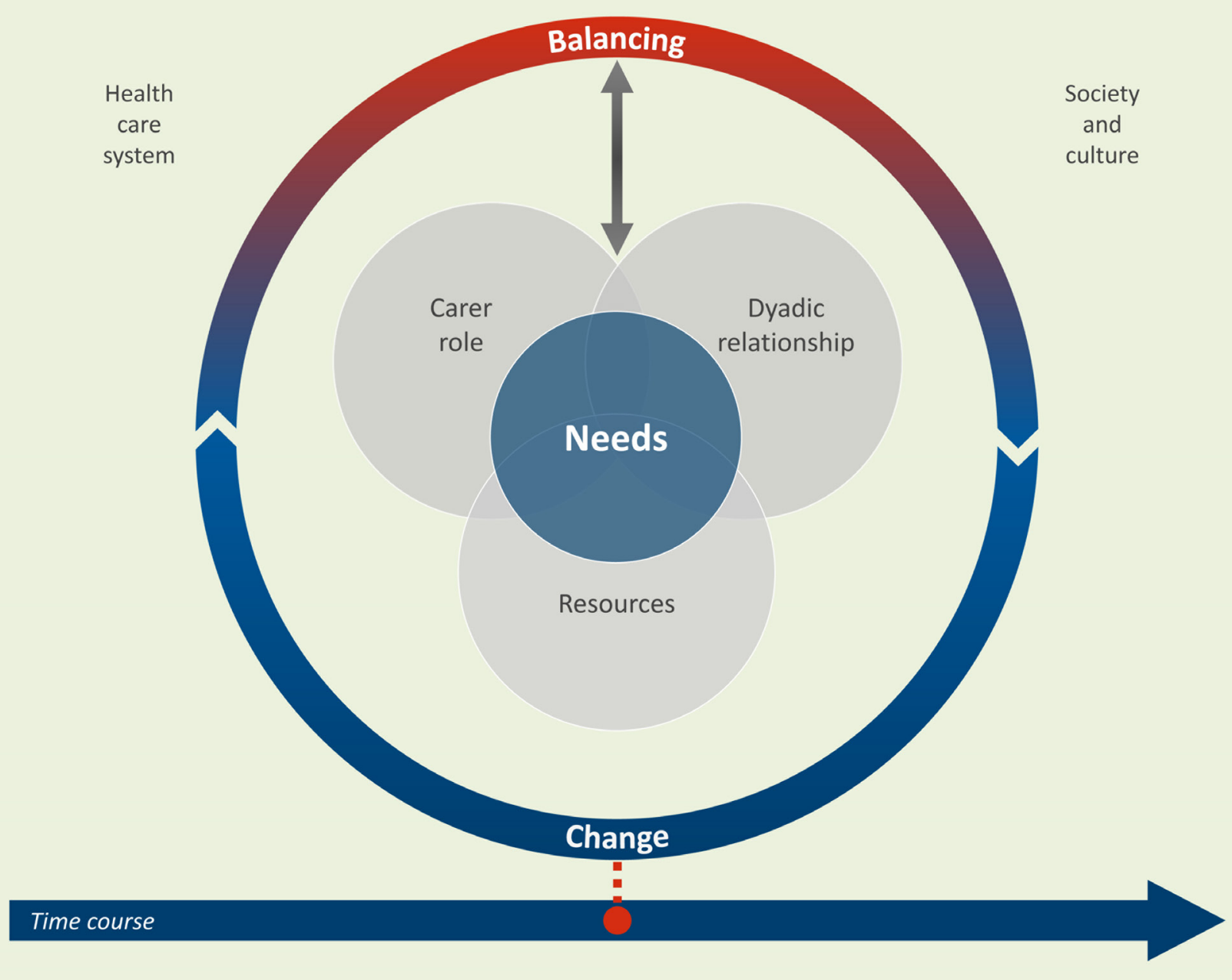

Figure 3 Conceptual model of stability of home-based care arrangements for people living with dementia.

introduce the concepts one by one. To ensure the readability of the results section, we decided to cite only one key reference for each argument. Online supplemental appendix D presents information (for each of the included publications) on the question if a publication contributed to our understanding of a specific concept of the developed middle-range theory.

\section{Change}

\section{Conceptualisation}

To a great extent, the trajectories of home-based dementia care arrangements are driven by change. One major change is the progression of dementia. Dementia is a neurodegenerative syndrome associated with a progressive deterioration of cognitive and physical functioning that increasingly challenges the person with dementia in managing his or her daily life independently. Consequently, the person with dementia often relies on the support of family members, friends, neighbours and professional service providers. The change caused by dementia introduces various additional challenges to the home-based care arrangement. In general, gradual changes can mostly be well balanced, whereas unexpected changes challenge the stability of the home-based care arrangement.

\section{Analyses and synthesis}

Table 1 provides an overview of the fields in which change occurs.

If change occurs, the informal carer appraises the change and decides whether and how he or she needs to balance the changed situation. The appraisal of change is highly individual as follows: some informal carers interpret the first cognitive symptoms of the person with dementia as a part of normal ageing, while others identify these changes as pathological and seek a diagnosis. ${ }^{41}$ During this phase, some dyads experience a period of uncertainty while coping with 'diagnostic shock', ${ }^{42}$ whereas others feel relieved by the final diagnosis. ${ }^{43}$ This appraisal of the impact and meaning of a specific change by the informal carer is as important as the change per se. $^{44}$

In the context of this middle-range theory, change is the most crucial if it challenges the stability of the homebased care arrangement and provokes crises that cannot be balanced. MacNeil Vroomen and colleagues ${ }^{45}$ built a 


\begin{tabular}{ll}
$\begin{array}{l}\text { Table } 1 \\
\text { meta-study sample }\end{array}$ \\
$\begin{array}{l}\text { Change } \\
\text { related to: }\end{array}$ & Fields of change \\
\hline $\begin{array}{l}\text { Person with } \\
\text { dementia }\end{array}$ & $\begin{array}{l}\text { Cognition, behaviour, (Instrumental) Activities } \\
\text { of Daily Living (ADL/IADL) functioning, } \\
\text { physical health, mental health and age }\end{array}$ \\
\hline Informal carer & $\begin{array}{l}\text { Physical health, mental health, ADL/IADL } \\
\text { functioning and age }\end{array}$ \\
Needs & $\begin{array}{l}\text { Needs of the person with dementia and } \\
\text { needs of the informal carer }\end{array}$ \\
Carer role & $\begin{array}{l}\text { Self-conception and external conception of } \\
\text { the carer role and having/assuming other } \\
\text { roles }\end{array}$ \\
\hline $\begin{array}{l}\text { Dyadic } \\
\text { relationship }\end{array}$ & $\begin{array}{l}\text { Roles in the dyad, reciprocity and } \\
\text { relationship quality }\end{array}$ \\
\hline Resources & $\begin{array}{l}\text { Practical skills, mental resources, financial } \\
\text { resources and arrangement of informal/ } \\
\text { formal networks }\end{array}$ \\
\hline $\begin{array}{l}\text { Society and } \\
\text { culture }\end{array}$ & $\begin{array}{l}\text { Policy strategy, societal discourse of } \\
\text { dementia, demographics and spatial } \\
\text { environment }\end{array}$ \\
system & $\begin{array}{l}\text { Policy strategy, availability and financing of } \\
\text { health and social care services }\end{array}$ \\
\hline
\end{tabular}

theory of crises in dementia care. Their conceptualisation of the dementia crisis process and the repeating alternation between imbalance and homeostasis is consistent with our understanding of stability.

Change can trigger positive or negative consequences. If the informal carer can accept the (unavoidable) changes in the person with dementia and the related changes in his or her own life ${ }^{46}$ and decide to acknowledge the positive aspects of his or her situation, this may result in supportive changes in his or her attitude and ultimately facilitate the finding of meaning. ${ }^{47}$

Change can be slow, developmental and predictable or rapid, situational and unexpected. ${ }^{48}$ Different forms of dementia or rather late or early onset of dementia, can result in different changes. ${ }^{49}$ In general, changes that can be foreseen (such as a progressive cognitive decline) are easier to balance than changes that are unexpected (such as a fall) ${ }^{15}$ Gradual changes can be monitored without time pressure, whereas in the face of a rapid change, informal carers are must act spontaneously. ${ }^{50}$ Some authors have concluded that serious and unexpected changes rather than constant changes are the initial triggers leading the informal carer to start thinking about the termination of home-based care ${ }^{51}$ Liken $^{52}$ reported that $80 \%$ of the informal carers in her sample moved their relatives with dementia to institutions due to unexpected events.

\section{Balancing}

\section{Conceptualisation}

Change in a current care situation triggers an action by the informal carer. The most prominent action strategy in the proposed middle-range theory is balancing. Informal carers strive to balance the situation to maintain the stability of their care arrangements. Balancing can either target internal processes (eg, changing mindset) or practical actions. Imbalance occurs if there is a mismatch between the acute challenge caused by a change and the informal carer's repertoire to adapt to this challenge. If the informal carer loses control and fails to balance the situation, the home-based care arrangement often moves into a crisis, followed by a period of instability.

\section{Analyses and synthesis}

We identified balancing as a process that is often divided into four steps. First, the informal carer appraises a change and reflects on whether he or she should adapt to the change. Thus, the informal carer weighs the risk of taking no action against the perceived benefit of adaptation. Second, the informal carer decides how to adapt to maintain the stability of the situation. Third, the informal carer acts with the intention to balance the care situation. Fourth, the informal carer evaluates whether his or her balancing was adequate to maintain the stability of the care arrangement. ${ }^{53}$

The notion of different types of informal carers who perform balancing in different ways was very prominent in our sample. In some studies, a distinction was made between informal carers who proactively plan ahead for the future and informal carers who delay decisions and actions until they obviously need to react in a crisis. ${ }^{54}$ Regarding the maintenance of stability of the home-based care arrangement, a proactive balancing strategy seems to promote stability, whereas a reactive attitude challenges stability. ${ }^{53}$

The following examples illustrate that the fields of actions targeted by the balancing of an informal carer are diverse; informal carers balance the needs of the person with dementia with their own needs to care for their physical and emotional well-being. ${ }^{55}$ Regarding the carer role, informal carers balance their mindsets; they accept the fact that their family members have dementia and choose to have a positive attitude, ${ }^{47}$ modify their beliefs and expectations ${ }^{56}$ and attempt to find peace of mind. ${ }^{46}$ Furthermore, informal carers balance the duties they face in their carer roles with their duties in other roles, such as being a parent or having a professional occupation. ${ }^{57}$ Informal carers balance the reciprocity in their dyadic relationships ${ }^{58}$ and the interactions within their informal ${ }^{59}$ and formal ${ }^{60}$ support networks and strive to establish collaborative partnerships. ${ }^{54}$ In this context, informal carers value their support networks as an essential source of the support needed to continue in their carer roles. ${ }^{61}$ In addition, they balance the challenge of caring for a person with dementia by developing expertise 
in their knowledge regarding dementia and their practical skills. ${ }^{43}$

Related to our understanding of a trajectory as a cyclic process of change and balancing over time, some publications in our sample contextualised their research with theories concerning chronic illness trajectories ${ }^{42}$ and conceptualised dementia care as occurring in phases over time.$^{62}$ Some studies shed light on informal carers' balancing throughout the whole care trajectory. ${ }^{63}$ However, overall, our sample strongly focused on the decision making of informal carers regarding the institutionalisation of the person with dementia. ${ }^{64}$ Therefore, in extent research, there is an emphasis on balancing during the phase of the trajectory in which instability is impending or already definite.

\section{Needs}

\section{Conceptualisation}

The needs of the person living with dementia and the informal carer occupy a central position in our conceptual model because a stable care arrangement can be created and maintained only if these needs are met. Over the trajectory of a home-based care arrangement, needs change, and continuous balancing is necessary. Conflicts due to the competing needs of one person or the divergent needs of both persons in the dyad can emerge and must be resolved to the greatest extent possible.

\section{Analyses and synthesis}

A prevailing assumption among the studies in the sample was that the stability of a care arrangement is at risk if the needs of the person with dementia or his or her informal carer are unmet. ${ }^{65}$

Regarding the person with dementia, the commonly stated needs were health-related needs or needs related to limitations in the activities of daily living. ${ }^{66}$ These needs were discussed as a consequence of the progression of dementia or ageing and multimorbidity in general. Regarding the informal carer, the identified needs were often related to the consequences of giving care, the informal carers' well-being ${ }^{67}$ or lack of resources. ${ }^{68}$ Needs related to the lack of resources often included needs for professional support services or educational programmes. ${ }^{50}$

Furthermore, during the analysis process, we identified numerous needs of the person with dementia and the informal carer that were related to one of the concepts of the characteristics of the care arrangement, such as the need to find meaning in the demanding carer role, ${ }^{47}$ the need to preserve reciprocity in the dyadic relationship ${ }^{46}$ and the need for resources in the form of a supporting network of family, friends, neighbours and professionals ${ }^{62}$ or specific services (such as care) ${ }^{69}$ According to these findings, needs represent a concept with a very large conceptual overlap with the other concepts and major influence; whether needs are met or unmet shapes the forming of the other concepts which, in turn, either promotes or challenges stability. Therefore, our conceptual model positions needs as the central concept.

As dementia progresses, the informal carer becomes increasingly responsible for anticipating the needs of the person with dementia. Considering the needs of the person with dementia and his or her own needs is not an easy task for the informal carer as these needs can be conflicting. ${ }^{52}$ Furthermore, the anticipated needs of the person with dementia could also conflict with each other, such as the need for autonomy versus the need for safety. ${ }^{70}$ Additionally, the informal carer could have conflicting needs, such as the need to be a dedicated carer and the need to care for himself or herself. Therefore, these conflicts in needs could be distinguished as interneed conflicts (ie, the persons in the dyad have conflicting needs) and intraneed conflicts (ie, the needs of one person conflict with each other).

\section{Carer role}

Conceptualisation

The way an informal carer forms his or her carer role has a crucial impact on the stability of home-based care arrangements. Becoming and being an informal carer can be perceived as a positive mission, an undesired obligation or a mélange of both positive and negative experiences. Regarding the stability of home-based care arrangements, how the informal carer assumes the carer role and manages to balance this role with other social roles seem to be important.

\section{Analyses and synthesis}

At the very beginning of the trajectory, assuming the carer role is a gradual and unconscious process. ${ }^{42}$ Subsequently, informal carers realise their actions and identify as carers. ${ }^{41}$ Adapting to the new role is often challenging ${ }^{43}$; for instance, because informal carers assume new responsibilities $^{71}$ and feel unprepared, ${ }^{60}$ adapting to the new role can be particularly challenging if the beginning of the care trajectory is unexpected. ${ }^{72}$

Assuming the carer role is usually motivated by the dyadic relationship between the informal carer and the person with dementia. ${ }^{70}$ In general, there are societal expectations to care for family members. These expectations vary across countries and cultures and are reflected in the respective healthcare systems. ${ }^{73}$

Informal carers have different feelings regarding their roles. For some informal carers, caring is a reciprocal act as follows ${ }^{41}$ : adult children often understand caring as an opportunity to give back to their parents ${ }^{47}$ and spouses often view caring as fulfilling their marriage vows. ${ }^{58}$ Being intrinsically motivated to provide care supports stability. ${ }^{55}$ A positive appraisal of giving care increases the motivation of the informal carer $^{70}$ and the likelihood that he or she will continue caring at home. ${ }^{74}$ Intrinsically motivated informal carers take pride in their roles and often experience personal growth. ${ }^{47}$ For other informal carers, 
assuming the carer role is considered a moral obligation. ${ }^{43}$ Such carers feel as if they have no choice ${ }^{52}$ either because no one else is available ${ }^{75}$ or others expect them to provide care. ${ }^{59}$ This situation can lead to feelings of entrapment and overload in the carer role. ${ }^{70}$ Informal carers who experience few if any positive aspects and feel burdened by their duties have a lower commitment to continue caring at home tend to develop a desire to institutionalise the person being cared for in the future $\mathrm{e}^{76}$ and actually end the home-based care arrangement sooner. ${ }^{77}$ Attitudes towards the carer role can change over the trajectory of the care arrangement, which was described in the studies in the sample as a modification of beliefs or personal expectations, ${ }^{56}$ a cognitive shift ${ }^{41}$ or a result of adapting and learning. ${ }^{42}$

A consequence of assuming the carer role may be role conflicts with other obligations in the life of the informal carer. ${ }^{57}$ These obligations can be a professional occupation, ${ }^{78}$ the informal carer's family life (especially among carers who have children) ${ }^{50}$ or other social interactions with friends or neighbours. ${ }^{79}$ In particular, adult children are concerned with conflicting obligations. ${ }^{53}$ If the informal carer does not successfully balance his or her social roles, the stability of the care arrangement could be endangered. ${ }^{80}$

\section{Dyadic relationship \\ Conceptualisation}

Caring occurs within relationships, and most informal carers perceive caring as a natural continuation of the dyadic relationship with the person living with dementia. Therefore, the structure of the dyadic relationship (ie, kinship relation, family status and living situation) and the quality of the dyadic relationship (ie, primarily the renegotiation of roles and the maintenance of reciprocity) impact the stability of the home-based care arrangement. The direction of the effect of the relationship quality on stability may reverse over the trajectory.

\section{Analyses and synthesis}

Most studies in our sample stated that close kinship relationships promote the stability of a home-based care arrangement, ${ }^{68}$ whereas distanced kinship relationships increase the likelihood of a prompt cessation of homebased care.$^{79}$ In general, spouses tend to continue to care at home the longest, ${ }^{81}$ children tend to end home-based care sooner than spouses ${ }^{82}$ and other informal carers tend to institutionalise sooner than children. ${ }^{83}$ Regarding the family status of the person with dementia, there is consensus that being married or living in a partnership supports whereas being single challenges the continuation of home-based care. ${ }^{84}$ The availability of at least one informal carer seems to be indispensable. ${ }^{85}$ Living in the same household as the informal carer is supportive of the maintenance of a stable situation at home, whereas living alone is often a challenge. ${ }^{86}$ These structural aspects of the dyadic relationship do not directly influence stability but rather function as indicators of the other concepts included in our middle-range theory. The kinship relationship, family status and living situation influence the quality of the dyadic relationship (eg, spousal affection vs filial piety), the self-construction of the carer role (eg, motives to provide care) and the availability of informal and formal resources (eg, having adult children).

A prevailing assumption among the studies in the sample was that a good past or present relationship quality supports the continuation of home-based care, whereas a low past or present relationship quality challenges stability. ${ }^{46}$ Over the trajectory, the forming of the dyadic relationship is influenced by changes in the person with dementia. In many chronic illness trajectories, dyadic relationships tend to develop an asymmetric structure, with the carer being dominant. These changes are especially meaningful in a dementia care trajectory as they usually lead to a shift in former roles and are often accompanied by a strong decline in the reciprocity of the dyadic relationship.

In the dementia care trajectory, premorbid roles within the dyadic relationship are questioned and adapted to the current situation. ${ }^{70}$ Sometimes, a swap of premorbid (often traditional and gender-related) roles occurs, for example, carer husbands perform household tasks, carer wives manage financial issues ${ }^{43}$ and children assume parentlike roles. ${ }^{87}$ Many informal carers perceive this process of adapting long-standing roles in their dyadic relationships as a challenging transition that may be accompanied by a decline in the quality of the dyadic relationship, which is often conceptualised as shrinking reciprocity.

In reciprocal relationships, informal carers have a high willingness to continue care at home. ${ }^{55}$ If informal carers appreciate the opportunity to reward the love and care they received in the past, ${ }^{47}$ succeed in maintaining affectionate bonds ${ }^{61}$ and continue engaging in activities and rituals that they previously enjoyed, ${ }^{58}$ they tend to value their dyadic relationships as reciprocal and find meaning in their carer roles. ${ }^{88}$ In contrast, if informal carers start to complain about a decline in the reciprocity of their dyadic relationships, they lost a major source for continuing care ${ }^{58}$ These informal carers report changes in their feelings towards their relatives with dementia, ${ }^{70}$ a decreased level of affection, ${ }^{89}$ emotional distance and estrangement ${ }^{41}$ and a perception of having fallen out of love $^{75}$ and ultimately describe the relationship with the relative with dementia as lost. ${ }^{70}$

Over the trajectory of a care arrangement, the direction of the effect of the dyadic relationship on the stability of the home-based care arrangement may change. At the onset and during the early phases of the trajectory, it is assumed that the closer the kinship relationship is and the higher the quality of the dyadic relationship is, the higher the chance that the home-based care arrangement will remain stable. However, at some point, the direction of this effect may reverse. In the long term, the intent to keep the person with dementia at home for as long as possible is often associated with the expense of the informal carer's physical and 
mental well-being or the health and safety of the person with dementia. ${ }^{90}$ If the informal carer does not anticipate the critical phase when he or she will no longer be able to balance the care situation adequately, he or she might miss the opportunity to ensure a smooth and well-planned termination of home-based care.

\section{Resources}

\section{Conceptualisation}

Resources are pivotal for informal carers to balance the consequences of constant change and create and maintain a stable care arrangement. The more resources available in general, the more available resources fit the dyad's needs, and the smarter these resources are mobilised, the better the stability of the care arrangement. The availability and use of resources change over the trajectory and can be differentiated as intrapersonal resources and interpersonal resources.

\section{Analyses and synthesis}

In many publications, various resources were studied to examine their impacts on stability. If informal carers judge their resources as inadequate to balance the situation, the stability of the care arrangement could be endangered. ${ }^{91}$

In the category of intrapersonal resources, mental resources (eg, resilience and related concepts, such as mastery, a sense of competence and self-efficacy) were the most common resources researched. ${ }^{92}$ Skills, such as hands-on care skills or communication skills, were the second most studied group of resources researched in the intrapersonal category. ${ }^{54}$ Furthermore, many studies focused on the education, income levels ${ }^{84}$ and the physical health ${ }^{45}$ of the informal carer.

In the category of interpersonal resources, the studies in our sample investigated social support (eg, family members, friends or neighbours) ${ }^{84}$ and formal support (eg, respite care, community nurses or meal services). ${ }^{15}$ Regarding interpersonal resources, notably, the overall amount of potential social or formal support is often less important than the quality of the relationships and interactions between the persons involved. ${ }^{89}$ Meaningful interactions and communication between the informal carer and his or her supporters is highly important for the creation of stable care arrangements. ${ }^{43}$

All resources are dynamic and may change during the trajectory of a home-based care arrangement. For instance, the informal carer may experience a decline in support from his or her social network and an increase in social isolation if family members and friends withdraw. ${ }^{42}$ In contrast, meaningful new relationships, for example, with the members of a support group, could grow over the trajectory. ${ }^{3}$ The possible positive development of resources, such as increased resilience or the learning of new skills, were discussed as gains of caring, ${ }^{70}$ while a decline in resources, such as deteriorating health or decreasing financial resources, were discussed as burdens that may endanger the stability of home-based care. ${ }^{93}$
One study in our sample adopted an explicit strengthbased perspective as its theoretical foundation and highlighted the meaning of resources in the context of positive outcomes of caregiving. ${ }^{3}$ Other studies explicitly referred to a stress model, for example, the stress process model $^{38}$ or the stress-coping model. ${ }^{94}$ In these stress models, resources play an important role in coping with challenging life events. Studies adopting a theoretical perspective focusing on social inequality in the allocation of resources were missing in our sample, which could be interpreted as the lack of a societal perspective and emphasis on the individual/dyadic perspective.

\section{Society and culture \\ Conceptualisation}

Home-based dementia care arrangements are influenced by the society and culture in which the persons with dementia and their informal carers live. Therefore, the prevailing values of a specific culture shape the stabilityrelated context.

\section{Analyses and synthesis}

Regarding cultural values, we included studies addressing the societal expectations of different cultures in different geographical regions, for example, China ${ }^{95}$ and Europe, ${ }^{96}$ and research addressing the societal expectations of different ethnic groups in one country, for example, Chinese people in Australia ${ }^{78}$ or Latinos in the USA. ${ }^{97}$ In some cultures and ethnic groups, prevailing traditional values lead people to prefer family care and refuse to involve professional support services. In such contexts, the included studies described 'caregiving as a fulfilment of cultural values ${ }^{, 97}$ or a 'powerful cultural tradition of family care' ${ }^{96}$ In a German study, von Kutzleben and colleagues ${ }^{98}$ identified the country of origin of the person living with dementia as a significant influencing factor as follows: informal carers who cared for a person with dementia with a migrant background perceived the care situation as more stable than informal carers caring for a care recipient who was native born. Mausbach and colleagues ${ }^{97}$ and Sun and colleagues ${ }^{99}$ reported that in the USA, Latinos and African-Americans tend to institutionalise their relatives later than their Caucasian counterparts.

In cultures bound to traditional values, (mostly female) family members are expected to assume the informal carer role. On the one hand, these informal carers often feel valued by their social networks and tend to have positive attitudes towards their carer roles. ${ }^{97}$ On the other hand, informal carers are frequently placed under pressure (by the care recipient him/herself or other family members) to fulfil the expected traditional roles regardless of the personal cost or consequences. Particularly if there are divergent notions of familial obligation between different family members and/or the informal carer refuses to accept the assigned traditional role, role conflicts and family disagreement emerge with possible consequences on the stability of the care arrangement. ${ }^{78}$ 
Regarding the cultural/societal context, distinctive changes in the near future are expected. The profound demographic changes, increasing number of elderly people, reduction in family size, geographic spread of family members and workplace participation (of women) could result in the older generation relying on fewer potential family caregivers and a smaller amount of informal resources than before. ${ }^{100}$ In relation to cultures still bound to traditional values, 'modern' families may no longer be able to maintain the traditional roles ${ }^{93}$ or may consciously shift from the traditional values held by older generations. $^{78}$

\section{Healthcare system \\ Conceptualisation}

The national policy agenda, the associated promotion of specific formal support services and the availability and financing of such services affect the informal carer's options to use or reject the use of formal services. In addition, the national policy context and the respective healthcare system are shaped by the perceived cultural and societal values. This context influences the stability of home-based care arrangements.

\section{Analyses and synthesis}

Dramé and colleagues ${ }^{100}$ identified the following universally accepted principles in all member countries of the Organisation for Economic Cooperation and Development: remaining at home for as long as possible, delaying institutionalisation, supporting informal carers and coordinating services at a local level whenever possible. However, how these principles are incorporated into national policy agendas and implemented in healthcare insurance systems and care practice widely differ across different cultures and countries worldwide. ${ }^{73}$ Some national policy agendas rely on extensive family participation (eg, Asia, eastern and southern Europe, Germany and the USA), while in other agendas, the additional integration of formal support services (including institutional care) is highly accepted (eg, Australia, Western Europe and Scandinavia). According to these diverse policy aims, how financial resources are governed and allocated varies, which often influences the decision of informal carers to continue or end home care. ${ }^{85}$

In our sample, there was a consensus that most homebased care arrangements require some type of formal support services at some point during the trajectory. ${ }^{60}$ Therefore, the use of formal support services may either function as a protective factor for stability because the unmet needs of the care arrangements are addressed or accelerate the termination of home-based care because positive experiences with the increasing integration of formal support services pave the way for the final decision to institutionalise the person with dementia. ${ }^{82}$ Informal carers particularly value education and training, ${ }^{50}$ case management, ${ }^{45}$ home care services, ${ }^{86}$ respite services, ${ }^{98}$ support groups, ${ }^{42}$ hospice services ${ }^{101}$ and financial aid ${ }^{81}$ to support the maintenance of a stable situation at home.
The setup of different healthcare systems and how formal services are promoted and funded seriously affect the availability and use of such services. Informal carers state a need for adequate financial aid. ${ }^{71}$ In the US context, Cepoiu-Martin and colleagues ${ }^{84}$ reported that a higher percentage of spending on in-home care services and a higher number of home health agencies decreased the risk of nursing home placement. In the Italian context, Sansoni and colleagues ${ }^{102}$ argued that the coverage of nursing home costs by the state could increase the rate of nursing home placement. Families who cannot afford to pay for a nursing home avoid placement and tend to continue care at home, ${ }^{51}$ but if the costs for institutional care are covered by the state, families with a lower income tend to institutionalise their relatives earlier, while families with a higher income more often continue homebased care as they can pay for community-based services out of pocket. ${ }^{103}$

In summary, the existence and availability of formal support services and adequate financial resources (either covered by a healthcare system or private means) allow informal carers to decide which formal support services support the maintenance of stability in their current situations.

\section{DISCUSSION}

In this meta-study, we developed a middle-range theory of the 'Stability of home-based care arrangements for people living with dementia' (SoCA-Dem theory) that emphasises the perspective of the informal carer. We conceptualised stability as a complex phenomenon comprising the following three components: (1) the trajectory of the home-based care arrangement comprising a cyclic process of change and balancing over time; (2) the characteristics of the care arrangement, including the concepts needs, carer role, dyadic relationship and resources; and (3) the context, including the concepts of society and culture and the healthcare system. Most concepts of the proposed middle-range theory have been well studied individually but have not been examined from an integrative perspective. The strength of this study and additional value of our new middle-range theory are that this study links the concepts to the phenomenon of stability and sheds light on the dynamics linking the components and concepts.

The following three ongoing debates in the scientific community became obvious in our meta-theory and metamethod analyses, influenced our conceptualisation of stability and allowed us to recognise some limitations: (1) the debate concerning predominant research focusing on dyads (mostly from the perspective of the informal carer) versus a more heterogeneous understanding of care arrangements; (2) the debate concerning the predominant stress/burden perspective in research focusing on informal care versus a framing of informal care that also considers positive aspects; and (3) the debate concerning the predominance of retrospective and cross-sectional 
studies versus the need for prospective and longitudinal studies.

1. As a consequence of the predominant focus of most studies in our sample on informal carers, our middlerange theory primarily reflects the perspective of one actor in the dyadic care arrangement and highlights the pivotal role of the informal carer in the creation and maintenance of stability. This emphasis on the informal carer can be interpreted as a limitation that, to some extent, may have been triggered by our search strategy in which the involvement of an informal carer in the care arrangement was chosen as an inclusion criterion. Although we were actually interested in the perspectives of all actors in dyadic care arrangements, the included publications nearly exclusively adopted the perspective of the informal carer and failed to provide insight into the perspectives of the person with dementia and other actors in the care arrangement. There is a vivid debate regarding this prevalent shortcoming in research concerning informal dementia care. For example, Ceci and colleagues ${ }^{104}$ criticised that the heterogeneity of care arrangements is often overlooked. We do not question that the person with dementia has a voice and plays an active role in the creation and maintenance of his or her care arrangement ${ }^{2105}$; we are aware that there is a growing population of people with dementia who live alone and need to cope without the help of an informal carer ${ }^{106}$; and we know that most home-based care arrangements rely on the support of formal service providers and have a triadic structure. ${ }^{107}$ Home-based care arrangements are often diverse "convoys of care ${ }^{108}$ and will likely become even more diverse in the future due to demographic shifts and changes in gender roles and family structures. In subsequent research, this middle-range theory should be extended to include diverse actors' perspectives regarding stability, ideally through participative research approaches. ${ }^{109} 110$ However, even in its present form, the SoCA-Dem theory is open to a heterogeneous conceptualisation of care arrangements as it already positions diverse informal and formal support networks as pivotal resources for the dyadic care arrangement. In addition, we assume that this middle-range theory may also be valid in the context of diverse chronic diseases. Currently, we hypothesise that our middle-range theory can be applied in future research focusing on more diverse care arrangements and other chronic diseases, but the components and concepts of the theory might change in their meaning and forming.

2. Many publications included in our sample highlighted the progression of dementia as the main driver of change leading to a growing care dependency of the person with dementia and increasing stress and burden for the informal carer. This assumption is mirrored by a high number of publications in our sample that (explicitly or implicitly) used a stress/burden framework as their theoretical perspective and therefore relied on a dominant but increasingly questioned research paradigm. ${ }^{111}$ Informal carer burden has often been identified as a main outcome to be studied and targeted in intervention studies, but it has been criticised that this approach usually leads to a mismatch between the simple research logic of 'identified problem - targeted outcome' and the individual and much more complex real-life experiences of dyads living at home. ${ }^{112}$ In-depth qualitative research focusing on the structure and underlying meanings of informal caring has been found to be very valuable in this respect. For example, von Kutzleben ${ }^{21}$ revealed that informal carers follow their own logics and strategies in response to change. Informal caring proved to be the expression of an individual informal care concept, which can be defined as the intrapersonal manifestation of motives, aims and personal boundaries. Considering these individual informal care concepts helps enhance our understanding of how informal carers perceive burden and that the reduction in burden is not necessarily the most important priority of all carers. The authors of the publications included in our meta-study had very diverse understandings of the phenomena of stress and burden. This conceptual ambiguity has also been critically discussed in the literature ${ }^{113}$ and in our case, it complicated a coherent interpretation of the study results regarding burden and its influence on stability. Although the stress/burden perspective was dominant in our sample, in some included studies, the nascent paradigm shift to a more multifaceted understanding of informal dementia care, including positive experiences, was apparent. ${ }^{5}$ The SoCA-Dem theory emphasises the coexistence of both positive and negative experiences; for meaningful interpretation, burden and positive experiences always need to be contextualised within the complex interplay of all theory components and concepts.

3. Several up-to-date phase models ${ }^{21} 114115$ emphasise constant change in the everyday lives of dyads living at home, followed by ongoing adaptation in a dynamic non-linear process. Our views are consistent with those described by von Kutzleben, ${ }^{21}$ who highlighted the importance of distinguishing between different phases of the dementia care trajectory as in each phase, a different type of change may affect stability in a specific way. According to this study, the middle stages of the trajectory during which the person with dementia is still quite actively involved in everyday life but already has difficulties mastering the activities of daily living independently might be perceived as particularly challenging with regard to the maintenance of stability. In some studies in our sample, the research was contextualised using theories concerning chronic illness trajectories ${ }^{19}$ and transitions, ${ }^{116}$ and 34 longitudinal studies showed that researchers valued following dyads over a certain time span. However, there are methodological challenges to assessing the development of trajectories, even in longitudinal research. The definite beginning of a trajectory is difficult to determine. Consequently, 
the studies in our sample largely included care arrangements in various and undefined phases within their individual trajectories and did not acknowledge this issue as a limitation. Furthermore, most studies focused on rather late phases of the trajectory, often on the phase during which a transition to an institution occurred. To generate deeper knowledge of stability in all phases of the trajectory, as recommended by Kralik and colleagues, ${ }^{117}$ there is a need for future longitudinal studies that provide valid assessments of the current stage of the trajectory of the individual care arrangement, ideally include care arrangements at the very beginning of their trajectories and follow such trajectories over a long period. In this type of longitudinal study, the focus should be on the specific forming of each concept of our middle-range theory during each phase of the trajectory and the influence of each concept on the creation of stability.

\section{Limitations}

This meta-study has limitations. Although we critically reflected and discussed its results with peer researchers, our theoretical perspective as well as our previous empirical research influenced the development of the SoCA-Dem theory. Theoretically, we conceptualised dementia care as a trajectory that is shaped by the interaction of individuals over time. ${ }^{19}{ }^{20}$ Empirically, the study by von Kutzleben ${ }^{21}$ identified the maintenance of stability as a leading principle of informal carers and shed light on this specific perspective. Furthermore, our working definition ${ }^{18}$ guided the deduction of the search strands and the preliminary coding scheme for this meta-study. Though the concepts of the new theory were created in an inductive and reflexive approach in the analysis phase, further research is needed to reduce existing bias within the SoCA-Dem theory. According to Roy, ${ }^{118}$ the circular relationship of theory to research to practice is particularly evident in middle-range theories. Their use in research and practice provides feedback for further theory development. ${ }^{25}$ Currently, we elaborate the interplay of the theory concepts on a descriptive level. We provide a middle-range theory that helps to organise and interpret the relationships of relevant concepts and that is rather constitutive than causal. ${ }^{26}$ To enhance the explanatory power of the SoCA-Dem theory, the causal relationships between the concepts need clarification beyond the current level. Another limitation concerns the dominance of research from Europe and North America in our sample. The possibility to reflect social and cultural differences was incorporated in the concept society and culture as well as in the concept healthcare system. Nevertheless, our SoCA-Dem theory might be influenced by a western perspective and therefore its applicability in other contexts needs to be explored. Finally, an additional limitation is grounded in the large number of included studies. To analyse and synthesise all these studies was time-consuming. For this reason, we did not perform the theoretical sampling as extensively as we planned according to the study protocol, ${ }^{27}$ and the time between the last database search and the publication of this meta-study is rather long.

\section{Conclusion}

This middle-range theory conceptualises the phenomenon of the stability of home-based care arrangements for people living with dementia. By adopting the perspective of the informal carer, the SoCA-Dem theory contributes to an enhanced understanding of the dynamic processes that contribute to stable or instable phases within the often long-lasting dementia care trajectory.

Elaborating on the perspective of the person with dementia is a necessary important goal for future research focusing on 'stability' and will enhance our understanding of the identified components and concepts. In addition to the aspired inclusion of the perspective of the person with dementia and other actors involved in home-based care arrangements, we highlight the need to further clarify the conceptual overlap and interplay of the theoretical components and concepts and the need to better understand the development of stability in all phases of the dementia care trajectory. All this will contribute to an ongoing improvement of the explanatory power of the SoCA-Dem theory and to its usefulness for scientists and healthcare practitioners. In the SoCA project, we address the current knowledge gaps in subsequent empirical research applying qualitative and quantitative methods. We aim to be able to distinguish between stable and unstable care arrangements and identify or develop interventions. Already, our middle-range theory can provide guidance for healthcare providers and policy makers: the concepts of the SoCA-Dem theory define topics that need to be considered to support the stability of homebased care arrangements. Support should be adapted to the individual forming of the concepts in the respective care arrangement. Effective interventions have to take into account the specific needs of the person living with dementia and the informal carer at a particular point of time.

Finally, we encourage the scientific community, policy makers and healthcare providers to use, reflect on and refine the SoCA-Dem theory and contribute to the ongoing discourse regarding stability to better enable dyads to live in the place of their choice currently and in the future.

Acknowledgements René Müller-Widmer, Saskia Kelinski, Christina Manietta and Jana Isabelle Braunwarth supported the author team in specific analyses and the layout of the tables and graphic material.

Contributors KK, JD, IH, MvK and BH substantially contributed to the conception and design of this meta-study, including the development of the research questions, search strategies, eligibility criteria, data extraction schemes and analytic procedures. KK, JD, IH and MvK performed the coding process. KK, JD and IH performed the data analysis and synthesis process. The proposed middle-range theory was repeatedly discussed, refined and finalised as a team by all authors. KK, JD and IH contributed to the draft of this manuscript. All authors read, provided feedback and approved the final manuscript.

Funding This meta-study was financed by the budget of the German Center for Neurodegenerative Diseases. Award/Grant number is not applicable. 
Competing interests None declared.

Patient consent for publication Not required.

Provenance and peer review Not commissioned; externally peer reviewed.

Data availability statement Data sharing not applicable as no datasets generated and/or analysed for this study. No data are available. No additional data available.

Supplemental material This content has been supplied by the author(s). It has not been vetted by BMJ Publishing Group Limited (BMJ) and may not have been peer-reviewed. Any opinions or recommendations discussed are solely those of the author(s) and are not endorsed by BMJ. BMJ disclaims all liability and responsibility arising from any reliance placed on the content. Where the content includes any translated material, BMJ does not warrant the accuracy and reliability of the translations (including but not limited to local regulations, clinical guidelines, terminology, drug names and drug dosages), and is not responsible for any error and/or omissions arising from translation and adaptation or otherwise.

Open access This is an open access article distributed in accordance with the Creative Commons Attribution Non Commercial (CC BY-NC 4.0) license, which permits others to distribute, remix, adapt, build upon this work non-commercially, and license their derivative works on different terms, provided the original work is properly cited, appropriate credit is given, any changes made indicated, and the use is non-commercial. See: http://creativecommons.org/licenses/by-nc/4.0/.

\section{ORCID iDs}

Kerstin Köhler http://orcid.org/0000-0002-8681-566X

Jan Dreyer http://orcid.org/0000-0002-9400-4787

Iris Hochgraeber http://orcid.org/0000-0003-1425-5955

Milena von Kutzleben http://orcid.org/0000-0002-8443-2593

Christiane Pinkert http://orcid.org/0000-0002-2692-1671

Martina Roes http://orcid.org/0000-0003-4531-8584

Bernhard Holle http://orcid.org/0000-0003-2549-7765

\section{REFERENCES}

1 World Health Organization. Dementia: a public health priority. Geneva: World Health Organization, 2012.

2 von Kutzleben M, Schmid W, Halek M, et al. Community-dwelling persons with dementia: what do they need? what do they demand? What do they do? A systematic review on the subjective experiences of persons with dementia. Aging Ment Health 2012;16:378-90.

3 Peacock S, Forbes D, Markle-Reid M, et al. The positive aspects of the caregiving journey with dementia: using a strengthsbased perspective to reveal opportunities. $J$ Appl Gerontol 2010;29:640-59.

4 Wadham O, Simpson J, Rust J, et al. Couples' shared experiences of dementia: a meta-synthesis of the impact upon relationships and couplehood. Aging Ment Health 2016;20:463-73.

5 DSF Y, Cheng S-T, Wang J. Unravelling positive aspects of caregiving in dementia: an integrative review of research literature. Int J Nurs Stud 2018;79:1-26.

6 Vasunilashorn S, Steinman BA, Liebig PS, et al. Aging in place: evolution of a research topic whose time has come. J Aging Res 2012;2012:120952

7 Dröes RM, Chattat R, Diaz A, et al. Social health and dementia: a European consensus on the operationalization of the concept and directions for research and practice. Aging Ment Health 2017:21:4-17.

8 Bartlett R, Brannelly T. Life at home for people with a dementia. Abingdon \& New York: Routledge, 2019.

9 Prince M, Wimo A, Guerchet M. World Alzheimer report 2015: the global impact of dementia: an analysis of prevalence, incidence, cost and trends. London: Alzheimer's Disease International, 2015.

10 Hajek A, Brettschneider C, Lange C, et al. Longitudinal predictors of institutionalization in old age. PLoS One 2015;10:e0144203.

11 Schaller S, Mauskopf J, Kriza C, et al. The main cost drivers in dementia: a systematic review. Int $J$ Geriatr Psychiatry 2015;30:111-29.

12 Vukoman M, Rüßler H. (Ehe-)Partnerschaft im Kontext der Pflegebedürftigkeit. Z Gerontol Geriatr2019.

13 Bundesministerium für Gesundheit. Zukunftswerkstatt Demenz. Berlin, 2017.

14 Toot S, Swinson T, Devine M, et al. Causes of nursing home placement for older people with dementia: a systematic review and meta-analysis. Int Psychogeriatr 2017;29:195-208.
15 Caron CD, Ducharme F, Griffith J. Deciding on institutionalization for a relative with dementia: the most difficult decision for caregivers. Can J Aging 2006;25:193-205.

16 Wiglesworth A, Mosqueda L, Mulnard R, et al. Screening for abuse and neglect of people with dementia. J Am Geriatr Soc 2010;58:493-500.

17 Wharton TC, Ford BK. What is known about dementia care recipient violence and aggression against caregivers? J Gerontol Soc Work 2014;57:460-77.

18 von Kutzleben M, Köhler K, Dreyer J. Stabilität von häuslichen Versorgungsarrangements für Menschen MIT Demenz. Entwicklung und Konsentierung einer definition von Stabilität durch Expertenfokusgruppen. Z Gerontol Geriatr 2017;50:210-8.

19 Corbin JM, Strauss A. Unending work and care: managing chronic illness at home. San Francisco: Jossey-Bass, 1988.

20 Nolan M, Grant G, Keady J. Understanding family care: a multidimensional model of caring and coping. Milton Keynes: Open University Press, 1996.

21 von Kutzleben M. Häusliche Versorgung von Menschen mit Demenz - Rekonstruktion des Versorgungshandelns informeller Versorgungspersonen im Zeitverlauf. Beltz Juventa, 2018.

22 Smith MJ, Liehr PR. Middle range theory for nursing, fourth edition. 4th edn. New York: Springer Publishing Company, 2018.

$23 \mathrm{E}-\mathrm{O} \mathrm{I}$. Theory development strategies for middle-range theories. ANS Adv Nurs Sci 2018;41:275-92.

24 Paterson BL, Thorne SE, Canam C. Meta-Study of qualitative health research. Thousand Oaks, London, New Delhi: Sage Publications, 2001.

25 Liehr P, Smith MJ. Middle range theory: a perspective on development and use. ANS Adv Nurs Sci 2017;40:51-63.

26 Risjord M. Middle-range theories as models: new criteria for analysis and evaluation. Nurs Philos 2019;20:e12225.

27 Dreyer J, Köhler K, Hochgraeber I, et al. Stability of home-based care arrangements for people living with dementia: protocol of a meta-study on mixed research. BMJ Open 2018;8:e021156.

28 Tong A, Flemming K, McInnes E, et al. Enhancing transparency in reporting the synthesis of qualitative research: ENTREQ. BMC Med Res Methodol 2012;12:181.

29 Boell SK, Cecez-Kecmanovic D. Literature reviews and the hermeneutic circle. AARL 2010;41:129-44.

30 Boell SK, Cecez-Kecmanovic D. A hermeneutic approach for conducting literature reviews and literature searches. CAIS 2014;34:257-86.

31 Finfgeld-Connett D, Johnson ED. Literature search strategies for conducting knowledge-building and theory-generating qualitative systematic reviews. J Adv Nurs 2013;69:194-204.

32 Bates MJ. The design of browsing and berrypicking techniques for the online search interface. Online Review 1989;13:407-24.

33 Dixon-Woods M, Kirk MD, Agarwal MS. Vulnerable groups and access to health care: a critical interpretive review. National coordinating centre NHS service delivery organ RD (NCCSDO), 2005.

34 Thomas J, Harden A. Methods for the thematic synthesis of qualitative research in systematic reviews. BMC Med Res Methodol $2008 ; 8$.

35 Pope C, Mays N, Popay J. Synthesising qualitative and quantitative health evidence. A guide to methods. McGraw-Hill Education, 2007.

36 Sandelowski M, Voils Cl, Barroso J. Defining and designing mixed research synthesis studies. Res Sch 2006;13:29.

37 Zarit SH, Reever KE, Bach-Peterson J. Relatives of the impaired elderly: correlates of feelings of burden. Gerontologist 1980;20:649-55.

38 Pearlin LI, Mullan JT, Semple SJ, et al. Caregiving and the stress process: an overview of concepts and their measures. Gerontologist 1990;30:583-94.

39 Aneshensel CS, Pearlin LI, Mullan JT. Profiles in caregiving: the unexpected career. Elsevier, 1995.

40 Corbin JM. The Corbin and Strauss chronic illness trajectory model: an update. Sch Inq Nurs Pract 1998;12:33-41.

41 Willoughby J, Keating N. Being in control: the process of caring for a relative with Alzheimer's disease. Qual Health Res 1991;1:27-50.

42 Lindgren CL. The caregiver career. Image 1993;25:214-9.

43 Frewer-Graumann S. Zwischen Fremdfürsorge und Selbstfürsorge: Familiale Unterstützungsarrangements von Menschen MIT Demenz und ihren Angehörigen. Wiesbaden: Springer, 2014.

44 de Vugt ME, Stevens F, Aalten P, et al. A prospective study of the effects of behavioral symptoms on the institutionalization of patients with dementia. Int Psychogeriatr 2005;17:577-89.

45 MacNeil Vroomen J, Bosmans JE, van Hout HP. Reviewing the definition of crisis in dementia care. BMC Geriatr 2013;13. 
46 Shim B. Finding meaning in the dementia caregiving relationship. (doctoral dissertation) Duke university 2011.

47 Lloyd J, Patterson T, Muers J. The positive aspects of caregiving in dementia: a critical review of the qualitative literature. Dementia 2016;15:1534-61.

48 Liken MA. Caregivers in crisis: moving a relative with Alzheimer's to assisted living. Clin Nurs Res 2001;10:52-68.

49 Bakker C, de Vugt ME, van Vliet D, et al. Predictors of the time to institutionalization in young- versus late-onset dementia: results from the needs in young onset dementia (NeedYD) study. J Am Med Dir Assoc 2013;14:248-53.

50 Toot S, Hoe J, Ledgerd R, et al. Causes of crises and appropriate interventions: the views of people with dementia, carers and healthcare professionals. Aging Ment Health 2013;17:328-35.

51 Schacke C, Zank S, Hausmann A. Pflegende Angehörige von demenziell Erkrankten : welche Faktoren beeinflussen den Transfer von der häuslichen in die stationäre Pflege? Psychother Psychosom Med Psychol 2012;62:367-74.

52 Liken MA. Critical incidents precipitating institutionalization of a relative with Alzheimer's. West J Nurs Res 2001;23:163-78.

53 Wackerbarth S. Modeling a dynamic decision process: supporting the decisions of caregivers of family members with dementia. Qual Health Res 1999;9:294-314.

54 Davis LL, Chestnutt D, Molloy M. Adapters, strugglers, and case managers: a typology of spouse caregivers. Qual Health Res 2014;24:1492-500.

55 Donovan ML. Edification of dementia caregiver uplifts characteristics. (doctoral dissertation) Capella university 2006.

56 Coe M, Neufeld A. Male caregivers' use of formal support. West $J$ Nurs Res 1999;21:568-88.

57 Schirra-Weirich L, Wiegelmann H. Typenbildung als Beitrag zur Weiterentwicklung von Versorgungsstrukturen für Menschen mit Demenz und ihren versorgenden Angehörigen. Ergebnisse einer Tandem-Studie im Rahmen des Modellprojekts "DemenzNetz Städte Region Aachen". In: Schäfer-Walkmann S, Traub F, eds. Evolution durch Vernetzung - Beiträge zur interdisziplinären Versorgungsforschung. Wiesbaden: Springer, 2017: 59-76.

58 Wuest J, Ericson PK, Stern PN. Becoming strangers: the changing family caregiving relationship in Alzheimer's disease. J Adv Nurs 1994;20:437-43.

59 Chang Y-P, Schneider JK, Sessanna L. Decisional conflict among Chinese family caregivers regarding nursing home placement of older adults with dementia. J Aging Stud 2011;25:436-44

60 Lethin C, Hallberg IR, Karlsson S, et al. Family caregivers experiences of formal care when caring for persons with dementia through the process of the disease. Scand J Caring Sci 2016;30:526-34.

61 Donovan ML, Corcoran MA. Description of dementia caregiver uplifts and implications for occupational therapy. Am J Occup Ther 2010;64:590-5

62 Daly L, McCarron M, Higgins A, et al. 'Sustaining place' - a grounded theory of how informal carers of people with dementia manage alterations to relationships within their social worlds. J Clin Nurs 2013;22:501-12.

63 Groen-van de Ven L, Smits C, Oldewarris K, et al. Decision trajectories in dementia care networks: decisions and related key events. Res Aging 2017;39:1039-71.

64 Ducharme F, Couture M, Lamontagne J. Decision-making process of family caregivers regarding placement of a cognitively impaired elderly relative. Home Health Care Serv Q 2012;31:197-218.

65 Schölzel-Dorenbos CJM, Meeuwsen EJ, Olde Rikkert MGM Integrating unmet needs into dementia health-related quality of life research and care: introduction of the hierarchy model of needs in dementia. Aging Ment Health 2010;14:113-9.

66 Gaugler JE, Kane RL, Kane RA, et al. Unmet care needs and key outcomes in dementia. J Am Geriatr Soc 2005;53:2098-105.

67 McLennon SM, Habermann B, Davis LL. Deciding to institutionalize: why do family members cease caregiving at home? J Neurosci Nurs 2010;42:95-103.

68 Gallagher D, Ni Mhaolain A, Crosby L, et al. Determinants of the desire to institutionalize in Alzheimer's caregivers. Am J Alzheimers Dis Other Demen 2011;26:205-11.

69 Glass AP. Family caregiving and the site of care: four narratives about end-of-life care for individuals with dementia. J Soc Work End Life Palliat Care 2016;12:23-46.

70 Quinn C, Clare L, Woods RT. Balancing needs: the role of motivations, meanings and relationship dynamics in the experience of informal caregivers of people with dementia. Dementia 2015;14:220-37.

71 Afram B, Verbeek H, Bleijlevens MHC, et al. Needs of informa caregivers during transition from home towards institutional care in dementia: a systematic review of qualitative studies. Int Psychogeriatr 2015;27:891-902.

72 Gaugler JE, Kane RL, Kane RA, et al. The longitudinal effects of early behavior problems in the dementia caregiving career. Psychol Aging 2005;20:100-16.

73 Afram B, Stephan A, Verbeek $\mathrm{H}$, et al. Reasons for institutionalization of people with dementia: informal caregiver reports from 8 European countries. J Am Med Dir Assoc 2014;15:108-16.

74 Kajiwara K, Nakatani H, Ono M, et al. Positive appraisal of in-home family caregivers of dementia patients as an influence on the continuation of caregiving. Psychogeriatrics 2015;15:26-31.

75 Lewis LF. Caregiving for a Loved one with dementia at the end of life. Am J Alzheimers Dis Other Demen 2015;30:488-96.

76 Cohen CA, Gold DP, Shulman KI, et al. Positive aspects in caregiving: an overlooked variable in research. Can J Aging 1994;13:378-91.

77 Gaugler JE, Yu F, Krichbaum K, et al. Predictors of nursing home admission for persons with dementia. Med Care 2009;47:191-8.

78 Caldwell L, Low L-F, Brodaty H. Caregivers' experience of the decision-making process for placing a person with dementia into a nursing home: comparing caregivers from Chinese ethnic minority with those from English-speaking backgrounds. Int Psychogeriatr 2014;26:413-24.

79 Gilhooly MLM. Senile dementia: Factors associated with caregivers preference for institutional care. Br J Med Psychol 1986;59:165-71.

80 Kwon S-H, Tae Y-S. Nursing home placement: the process of decision making and adaptation among adult children caregivers of demented parents in Korea. Asian Nurs Res 2012;6:143-51.

81 Luppa M, Luck T, Brähler E, et al. Prediction of Institutionalisation in dementia. Dement Geriatr Cogn Disord 2008:26:65-78

82 Runte R. Predictors of institutionalization in people with dementia: a survey linked with administrative data. Aging Clin Exp Res 2018;30:35-43.

83 Ebly EM, Hogan DB, Rockwood K. Living alone with dementia. Dement Geriatr Cogn Disord 1999;10:541-8.

84 Cepoiu-Martin M, Tam-Tham H, Patten S, et al. Predictors of long-term care placement in persons with dementia: a systematic review and meta-analysis. Int J Geriatr Psychiatry 2016;31:1151-71.

85 Saks K, Tiit E-M, Verbeek H, et al. Most appropriate placement for people with dementia: individual experts' vs. expert groups' decisions in eight European countries. J Adv Nurs 2015;71:1363-77.

86 Ledgerd R, Hoe J, Hoare Z, et al. Identifying the causes, prevention and management of crises in dementia. An online survey of stakeholders. Int J Geriatr Psychiatry 2016;31:638-47.

87 Redfern S, Norman I, Briggs K, et al. Care at home for people with dementia: routines, control and care goals. QAOA 2002;3:12-23.

88 Hirschfeld M. Home care versus institutionalization: family caregiving and senile brain disease. Int J Nurs Stud 2003;40:463-9.

89 Spitznagel MB, Tremont G, Davis JD, et al. Psychosocial predictors of dementia caregiver desire to institutionalize: caregiver, care recipient, and family relationship factors. J Geriatr Psychiatry Neurol 2006;19:16-20.

90 Kraijo H, de Leeuw R, Schrijvers G. How spouses evaluate nursing home placement of their demented partner: a study about the end of perseverance time. Scand J Caring Sci 2015;29:101-9.

91 Schacke C, Zank S. Zur familiären Pflege demenzkranker Menschen: die differentielle Bedeutung spezifischer Belastungsdimensionen für das Wohlbefinden Der Pflegenden und die Stabilität Der häuslichen Pflegesituation. Z Gerontol Geriatr 1998;31:355-61.

92 Gaugler JE, Kane RL, Newcomer R. Resilience and transitions from dementia caregiving. J Gerontol B Psychol Sci Soc Sci 2007;62:P38-44.

93 Chang Y-P, Schneider JK. Decision-making process of nursing home placement among Chinese family caregivers. Perspect Psychiatr Care 2010;46:108-18

94 Lazarus RS, Folkman S. Stress, appraisal, and coping. New York: Springer Publishing Company, 1984

95 Cheng S-T, Mak EPM, Lau RWL. Voices of Alzheimer caregivers on positive aspects of caregiving. Gerontologist 2016;56:451-60.

96 Verbeek H, Meyer G, Challis D, et al. Inter-country exploration of factors associated with admission to long-term institutional dementia care: evidence from the RightTimePlaceCare study. J Adv Nurs 2015;71:1338-50.

97 Mausbach BT, Coon DW, Depp C, et al. Ethnicity and time to institutionalization of dementia patients: a comparison of Latina and Caucasian female family caregivers. J Am Geriatr Soc 2004:52:1077-84. 
98 von Kutzleben M, Reuther S, Dortmann O, et al. Care arrangements for community-dwelling people with dementia in Germany as perceived by informal carers - a cross-sectional pilot survey in a provincial-rural setting. Health Soc Care Community 2016;24:283-96.

99 Sun F, Durkin DW, Hilgeman MM, et al. Predicting desire for institutional placement among racially diverse dementia family caregivers: the role of quality of care. Gerontologist 2013;53:418-29.

100 Dramé M, Lang P-O, Jolly D, et al. Nursing home admission in elderly subjects with dementia: predictive factors and future challenges. J Am Med Dir Assoc 2012;13:83.e17-20.

101 Badrakalimuthu V, Barclay S. Do people with dementia die at their preferred location of death? A systematic literature review and narrative synthesis. Age Ageing 2014;43:13-19.

102 Sansoni J, Anderson KH, Varona LM. Caregivers of Alzheimer's patients and factors influencing institutionalization of loved ones: some considerations on existing literature. Ann lg 2013;25:235-46.

103 Mittelman MSet al. A family intervention to delay nursing home placement of patients with Alzheimer disease. JAMA 1996;276:1725-31.

104 Ceci C, Symonds Brown H, Purkis ME. Seeing the collective: family arrangements for care at home for older people with dementia. Ageing Soc 2019;39:1200-18.

105 Read ST, Toye C, Wynaden D. The participation of people with dementia in the planning of their care and support: an integrative literature review. Dementia 2018

106 Eichler T, Hoffmann W, Hertel J, et al. Living alone with dementia: prevalence, correlates and the utilization of health and nursing care services. JAD 2016;52:619-29.
107 Quinn C, Clare L, McGuinness T, et al. Negotiating the balance: the triadic relationship between spousal caregivers, people with dementia and Admiral nurses. Dementia 2013;12:588-605.

108 Kemp CL, Ball MM, Perkins MM. Convoys of care: theorizing intersections of formal and informal care. J Aging Stud 2013;27:15-29.

109 Gove D, Diaz-Ponce A, Georges J, et al. Alzheimer Europe's position on involving people with dementia in research through PPI (patient and public involvement). Aging Ment Health 2018;22:723-9.

110 Bethell J, Commisso E, Rostad HM, et al. Patient engagement in research related to dementia: a scoping review. Dementia 2018;17:944-75.

111 Purkis ME, Ceci C. Problematising care burden research. Ageing Soc 2015;35:1410-28.

112 Ceci $\mathrm{C}$, Symonds Brown $\mathrm{H}$, Judge $\mathrm{H}$. Rethinking the assumptions of intervention research concerned with care at home for people with dementia. Dementia 2018.

113 Bastawrous M. Caregiver burden - A critical discussion. Int J Nurs Stud 2013;50:431-41.

114 Esandi N, Nolan M, Alfaro C, et al. Keeping things in balance: family experiences of living with alzheimer's disease. Gerontologist 2018;58:e56-67.

115 Clemmensen TH, Busted LM, Søborg J, et al. The family's experience and perception of phases and roles in the progression of dementia: An explorative, interview-based study. Dementia 2019;18:490-513.

116 Meleis Al, Sawyer LM, Im EO, et al. Experiencing transitions: an emerging middle-range theory. ANS Adv Nurs Sci 2000;23:12-28.

117 Kralik D, Visentin K, van Loon A. Transition: a literature review. J Adv Nurs 2006;55:320-9.

118 Roy C. Generating middle range theory. from evidence to practice. New York: Springer, 2014. 